\title{
Addiction Science for Professionals Working in Clinical Settings
}

\author{
Richard Pates and Roman Gabrhelík
}

\section{Introduction}

This chapter is aimed at doctors, psychologists, social workers, therapists, and other staff in the health sector, social care sector, and criminal justice system (e.g., prisons, probation) working in addiction. It is also written for workers in the nongovernmental (non-statutory or "third") sector with some professional training or expertise. These clinical workers often are the first to identify new trends in substance use, effects, problematic consequences, and problems that may support or hinder rehabilitation. Therefore, clinicians can play an important role in research. In many developing countries or in countries without a history of alcohol and other drug research, clinicians may be the only people who are able to document problems. At the same time, they also have a duty to identify and collect this information and distribute it. This chapter will discuss what sort of research might be suitable for clinicians, how to approach it, where to publish, and pitfalls in addiction research and publishing. The purpose is to encourage professionals who work in the field of addiction, not primarily as researchers, but as clinicians who have conducted work or research projects that could be worthy of publication. This chapter also provides instruction on how clinicians can collaborate with researchers.

Historically, clinicians have played an important role in research. It is worth remembering that the early pioneers in alcohol and other drug research were often doctors such as Trotter, Rush, and Huss (in alcohol research) as well as Dole and Nyswander (in research on the use of methadone in the treatment of

\section{How to cite this book chapter:}

Pates, R and Gabrhelík, R. 2017. Addiction Science for Professionals Working in Clinical Settings. In: Babor, T F, Stenius, K, Pates, R, Miovský, M, O’Reilly, J and Candon, P. (eds.) Publishing Addiction Science: A Guide for the Perplexed, Pp. 119-131. London: Ubiquity Press. DOI: https://doi.org/10.5334/bbd.f. License: CC-BY 4.0. 
heroin addiction). It is also of note that, today, many of the people working at the top of large research institutes and public health bodies such as the World Health Organization have clinical backgrounds in psychology and medicine.

Although a research component is included in many (or most) undergraduate and postgraduate clinical courses, it is sometimes seen as a process that must be passed before qualification rather than as an exciting opportunity to expand a professional role. In many professional fields, the number of welltrained staff who never do research or publish anything after they have qualified is surprising (e.g., Jowett et al., 2000; Salmon et al., 2007), especially given that the work clinicians perform, whether in the statutory or non-statutory sector, is usually based (or should be based) on proven results and methods founded on research-related best practices.

This lack of willingness to undertake research or to publish research results may result from lack of confidence, opportunity, or willing collaborators. But as will be seen in this chapter, there are plenty of opportunities and subjects appropriate to study systematically in the clinical setting. Although this chapter is not meant to teach research methods, it is aimed at those who have previously had some research training and who have had the opportunity to undertake research projects. It is also aimed at those interested in evaluating their work or investigating some aspect of their work that may be worthy of publication.

We cannot take for granted that the majority of professionals have the skills for conducting scientifically sound studies. To conduct a research study using appropriate design, adequate measures, and correct statistics can sometimes be difficult. Further, there are additional problems of trying to publish the findings in peer-reviewed journals.

Early addiction practice was based on a clinical approach (problems were observed, described, and explained), and this slowly started to shift toward empiricism (allowing for testing hypotheses through observation and experiment). More recently, an evidence-based approach to addiction services has been promoted and widely accepted. Evidence based practice (or applied addiction science) means that the nature and method of addiction services is based on findings from research studies. The level and quality of clinical work is quantified. Quantified results serve as an evidence of effectiveness or ineffectiveness of any interventions provided. ${ }^{1}$ In practice, this means a range of things, including treatment of addiction problems, prevention of relapse, and provision of aftercare and other post treatment interventions aimed at helping those in recovery get back to a regular lifestyle. Over time, addiction professionals began to ask questions about the effectiveness of the methods being used in the treatment of addiction problems, the prevention of relapse, and the provision of aftercare and other post treatment interventions aimed at reintegrating the person into daily life. As a consequence, interest in appropriate interventions grew. Professionals from the field started to search for new ways to achieve better results in less time but with a longer duration of action. 
Studying the effective factors in addiction services and monitoring the benefits of different interventions became the domain of research.

\section{Why bother Doing Research?}

For many clinicians, the idea of undertaking research may seem to be yet another demand on their time and not part of their job. But clinicians should always be asking whether what they do is effective and the best practice. As will be discussed later, research can take many forms in terms of evaluating interventions, trying to find the cause of a problem, studying individual cases or reviews of a subject area. Many of these areas may be too complex and involved for the professionals in clinical practice to undertake, but there are some types of research that are well within the capability of clinical staff. Examples of this are research into brief interventions with alcohol and tobacco, which has had an impact on clinical work.

Many benefits can be derived from taking part in research. There is an intrinsic satisfaction in undertaking a good piece of research, especially if it produces results that may affect your work and make it more effective. There is also the respect that you will earn from your colleagues. But most importantly, performing research can help to further your career. Even if your work has been mainly clinical, having publications on your résumé or curriculum vitae will do no harm and will probably enhance your career. Future employers will respect your endeavors into research.

\section{What Research is Appropriate for You?}

Choosing a research project that is suitable is very different if you are working in a clinical field rather than in an academic institution. In a clinical field, you will need to choose a research subject that permits access to participants (if it is a person-based project) and something that is manageable in the context in which you are working. Many clinical services perform regular audits of their work, and these are already simple forms of research. Of course, if this type of research is undertaken, it needs to be more rigorous than a standard audit and should conform to a research protocol.

Sometimes research questions may come from your search for a solution to a problem-you find that little work has been published in that subject area. In the 1990s, when the first author (R.P.) wanted to find treatments for compulsive injecting (needle fixation), a literature search revealed just one article published 20 years before that described three cases. This nevertheless led to a number of research collaborations in a clinical setting in which the problem was studied and psychological theories and treatment options were developed (Pates \& Gray, 2009; Pates et al., 2001). 
If the work you do routinely is common practice and already described in the literature, then it is unlikely to be of interest to journals. However, if you are doing something innovative or have noticed unusual results, this may well be worth formalizing and investigating. If you are planning innovative work, this should be investigated carefully following proper designs and ethical considerations.

The late Griffith Edwards, a great champion of addiction science and someone who was influential in encouraging junior researchers to publish their work, made an interesting observation that many clinicians will recognize. In a book of his to be published posthumously (Edwards, in preparation), he asked the question of where addiction research ideas came from. He observed that clinical research often comes from something said by a patient but also noted that the clinician "must have ears with which to listen. It is often too easy to ignore what patients may be saying by believing that expertise lies with the expert! He went on to describe a situation in which a patient of his commented that he (Edwards) had previously given the patient very bad advice: Edwards had told the patient that, to become sober, the patient would need a lengthy in-patient stay-the current practice at that time. The patient said he did not need that sort of help, would not accept it, and that it would mean the end of his business if he chose that path.

Inspired by this man, Edwards went on to conduct a comparison trial of in-patient versus out-patient treatment and found, at the 12-month followup, there was no significant difference between the two groups. This evidence helped to overturn the conventional consensus at the time-that in-patient treatment for a significant drinking problem is essential for recovery. This is a good example not only of the need to listen to patients but also of the need to challenge conventional ideas in places where they may be rigidly held.

In additional to quantitative reports, some journals will accept case reports or series of case studies (see also Chapter 8 on qualitative research), in which unusual findings may be reported (e.g., uncommon manifestation of diseases, "off-label" uses of medication, previously unreported effects of medications, unexpected effects of treatment). These studies can be of great interest because you may be the first to report a phenomenon-only make sure you are seeing and understanding cause and effect. These can add to the literature in an incremental but important way. It is often in clinical settings that these unusual practices come to notice, which could be the beginnings of a phenomenon or just unusual outliers in the field.

In addition to working within a centre there is the opportunity to work with other professionals doing similar work. This might entail being part of a multicentre trial, in which a number of treatment centres work on the same project to increase the numbers of people being treated and provide greater statistical power to the analyses. A multi-centre trial also allows for comparison across sites and thus increases the generalizability of findings. This sort of trial is usually expensive because it needs coordination, usually from a research center. 
This can be exciting work but requires a lot of extra effort to ensure that the interventions are the same in each center and that all the protocols are being followed in the same way.

Another type of investigation clinicians can do is historical research conducted by extracting data from case notes. For example, the first author of this chapter (R.P.) wanted to examine whether outcomes had changed in the clinic in which he was working from the establishment of the service 20 years previously to the present. This was performed by asking a number of questions that he formulated based on case notes and by taking a cohort of the first 200 patients registered with the clinic to establish things such as morbidity, mortality, recovery, and loss to the service. These data were then compared with data obtained from another cohort some 15 years later. This study evaluated a span of time when changes in practice were occurring in service delivery, and it was important to see if outcomes had improved. The study results actually had important consequences in terms of delivering services and learning lessons from practices that were found to be too rigid.

Good quantitative research is worth pursuing if the topic is original and not just repeating previous research. But, of course, many topics that have been researched are the product of an original idea that was investigated and then later research added to the findings and expanded it. In this way, individual studies become a body of research. Sometimes it is worth investigating a previously published research topic by adding a new dimension or helping to generalize a finding through the study of a different group. It must be borne in mind that, if the study is using a control group for comparison, it would be unethical to withhold a recognized treatment from the control group, even in the interests of the research.

Qualitative research is becoming more common in the addiction field. Twenty-five years ago, it was difficult to get qualitative research published because it was often not seen as "proper" research. That view has changed, and qualitative research is becoming more common. The advantage of conducting qualitative research is that you can investigate questions more deeply and follow up information that comes out of the research. It is often undertaken with fewer participants than quantitative research but still requires a rigid methodology and the same safeguards. (See Chapter 8 in this book for a full discussion on carrying out qualitative research.)

One major difference between working in a clinical setting and undertaking academic research is that, often in randomised controlled trials, there is a set of exclusion criteria that is used to remove what may be confounding factors for research. The problem for clinicians is that the people they treat are not subject to exclusion criteria. Storbjörk (2014) has written about this in a large piece of research on alcohol problems with 1,125 participants. She asked the following question: If 10 of the most common exclusion criteria were operationalized and applied to this group, what would be the percentage of real-world problem alcohol users excluded from her study and how would this exclusion, bias 
treatment outcomes. She found that $96 \%$ would have been excluded by at least one exclusion criterion. She found that on average, participants fulfilled 2.56 of the less exclusive criteria (eg unemployed or homeless) and 3.99 of the more exclusive criteria (Currently medicated for psychiatric problems or overdose recently). The percentage of treatment seekers excluded because of not meeting the less exclusive individual criteria ranged from 5\% being excluded for lack of education to $80 \%$ excluded for past or current addiction treatment. The importance of these results is that if our clinical work with real-world populations is informed by biased results, we will not see the same clear results that are published in some academic journals.

One example of this is in research undertaken in the United Kingdom on the treatment of amphetamine problems by substitute prescribing. This is now a common practice in the United Kingdom. However, one of the exclusion criteria has always been the presence of comorbid mental health problems, specifically because heavy use of stimulants such as amphetamine can produce paranoia and psychosis. Carnwath and colleagues (2002) challenged this by a piece of retrospective research examining the case notes of eight patients with schizophrenia who had been prescribed dexamphetamine for co-existing amphetamine dependence. The authors commented that the patients with coexisting problems had poorer treatment outcomes, often did not comply with treatment plans, and had frequent periods of hospitalization. However, they found that, in four of the eight cases examined, the prescription of dexamphetamine led to good progress in terms of both substance use and mental health. In two cases, progress was more equivocal although there had been some benefit, and two cases were deemed to be treatment failures but the condition of the patients was no worse at the end of treatment than at beginning. There was greater adherence to neuroleptic regimes, and none of the patients suffered an exacerbation of their psychotic symptoms as a result of treatment. This is an example of where exclusion criteria for being part of the trial were ignored and good results followed.

It is also true that, although randomised controlled trials are seen as the "gold standard" for research, use of a randomized controlled trial sometimes may be unethical if it means depriving one group of potentially advantageous treatment. An example of this can be seen in a research design in which needle exchanges are established in one city and not in another to measure the incidence of new viral infections among injection drug users. This, of course, would be entirely unethical and would have other methodological problems, unless there were only enough resources to establish programs in one of the cities.

\section{How to get Started}

Before starting on a project, you should discuss it with other colleagues to get their approval and cooperation. If this is seen to be feasible, then a thorough 
research protocol should be written with a description of the scientific need for the study (a literature search and an explanation of your hypothesis), methods of recruiting your sample of participants, methods of measurement, intervention, and statistical analysis.

If you have any doubts or questions, discuss them with colleagues or other people who are active in the field that you wish to conduct research in. Moreexperienced colleagues are often interested in what you might be doing and will be happy to answer questions and make suggestions about your line of research. Establish a coordinating committee that can provide advice and discuss the project as it progresses. This committee can include members of your department, but it is often useful to have someone from outside to ask the awkward questions and raise points you might not have thought about before. Another option might be to seek collaboration with doctoral students and postdoctoral students. Doctoral students and postdocs may offer their time, knowledge, and skills while supervised by their mentors.

Always make sure all the staff involved in the unit are aware of the research, understand the process, and have any queries answered satisfactorily. These may be the people who refer suitable subjects for your research or whose cooperation you may need to get to the project running smoothly.

Any research that involves human or animal subjects will require ethical approval. Where to obtain this will vary from country to country, but usually universities or major health centers will have a standing ethics committee. An application to the ethics committee will have to follow its standards and will possibly involve a personal appearance in front of the committee during which you will answer questions, provide assurances, and discuss potential changes to the research protocol.

Research usually requires extra funding. Such funds may be obtained as research grants, obtained as small grants from the employing authority, or absorbed in the normal running costs of the unit. Some research may be conducted in house with no extra costs by putting in place research protocols that allow other staff and colleagues to know what is being done. You will still need to be thorough and objective in your research, but it can be undertaken as part of clinical work. Investigators working in academic institutions will routinely be applying for research grants and will know the main funding bodies available in their field. These are likely to be less familiar to clinicians, but research funds are available from small charitable bodies as well as national funding bodies (e.g., the U.S. National Institute on Drug Abuse) and major organizations (e.g., the Gates foundation) who have a huge commitment to solving major world social and health problems. To be approved, it is important that you are working in an area covered by the funding body's activities and that, when you complete the application form, you answer all the questions and explain exactly what you are doing and why.

Make sure you have identified someone experienced in statistics who may be able to guide you on statistical techniques. Collaboration with a statistician 
from the beginning, when writing the project proposal, is encouraged (e.g., when focusing on patients, power sample analyses should be calculated before conducting research or when choosing appropriate data-collection tools). This is also true for someone experienced with quantitative methods when conducting qualitative research

As an example, an on-going project in the Czech Republic was conducted in therapeutic communities for users of illicit drugs. Research activities are relatively infrequent in these facilities because of many contextual reasons (e.g., low capacity of individual facility, low interest by staff, no uniform treatment models). Within last few years, a new, interesting research problem has emerged (not only) in the context of therapeutic communities: attention-deficit/hyperactivity disorder as a comorbid factor and risk factor for significantly higher treatment drop-out and reduction of treatment effect (Miovský et al., 2014). This interesting and important issue was formulated and clarified through a systematic discussion and series of meetings with staff within a two-year preparatory phase. The Czech team decided to invite the National Association of NGOs and its working group of therapeutic communities to participate. After a selection procedure, they contracted particular therapeutic communities, trained the staff in data-collection methods, and supervised the data-collection procedure. Particular communities were direct partners of the study and had participated since the beginning. To stay within the study budget and make the study manageable, however, the original concept had to remain limited because of potential travel costs and technical complications linked to the difficulty of testing all new clients for attention-deficit/hyperactivity disorder (which is an unpredictable and irregular procedure). Nonetheless, it is also a good example of how to create, through networking, a very attractive opportunity for extensive and sophisticated clinical research with a large number of clients.

\section{Who should be in My Article-Writing Team?}

Conducting a good-quality research project requires knowledge, skills, and enthusiasm combined with high levels of persistence. Writing a scientific article is, however, a discipline on its own. Many colleagues who are involved in the data-collection phase of your research will not participate in the actual writing of the article (s) for various reasons (e.g., because of a low interest in writing, lack of confidence or time). It is often the case that data are available but that there are only a limited number of people who are willing to write an article based on it. You may end up writing the actual manuscript on your own. To avoid this situation, you may want to start an early search for collaborators who will help you to write and submit articles to save time.

In the previous section, we suggested that a statistician be part of your research project. With the advent of modern statistical packages for your computer, it is often simple to run the statistics, but frequently people are using 
inappropriate statistics for the problem. It is important to get this right before you start. When your article is reviewed, your statistical techniques will be examined. If you have used the wrong technique, the article will be rejected. This will either mean you have wasted much time and effort or that it will take a lot more time to rework the statistics - which may of course then produce different outcomes. Similarly, preparing a high-quality qualitative article is difficult without the appropriate experience of a good qualitative researcher.

\section{Where Should I Publish?}

Choosing the right journal to which you may submit your finished article should be done with care. Chapter 3 of this book discusses this and should be consulted. There are many journals that focus specifically on addiction, but, in addition to these "addiction specialty journals," scientists and practitioners who work in the field also have a "mother" profession or discipline in which they have been trained (e.g., medicine, psychology). These disciplines also publish many journals in their fields, and these journals may publish articles on addiction. There are also journals published in countries in which information may be more local and more relevant to national or local populations. Therefore, there is a wide variety of potential journals to which you may submit your manuscript.

You must consider, therefore, whether the subject is of national or international importance. If the subject is mainly of interest to people in your country, it may be more appropriate to submit to a national journal. International journals may judge whether an article is of international interest and may not accept an article that is more local. However, it may be that a subject that appears to be local in scope becomes of interest to experts in many another parts of the world. Addiction is a worldwide problem, and practices spread. One example of this is that the use of water pipes to smoke tobacco is very common in the Middle East. Therefore, this form of substance use may be seen to be local. However, the practice does have great potential health risks, and the effects of the diaspora of refugees from this region to many other parts of the world will also export this practice and the concomitant health risks. Both authors and editors need to bear this sort of situation mind.

Before submitting, check the impact factor and acceptance rate of the journal. This can be found in Chapter 3, Table 3.1, in this book. Typically, journals with higher impact factors have lower acceptance rates. If you are submitting to a high-impact-factor journal, it will be more difficult for you to get your article accepted unless it is of high originality and good-quality science.

Furthermore, check the instructions for authors either in the relevant journal or on the website to ensure that the journal accepts the type of work your article describes. Make sure when you submit your article it conforms to the standards of the journal. Follow the instructions for authors regarding word length, style of referencing, and formatting of tables and figures. 


\section{What are the Pitfalls to Doing Research in this Setting?}

There are many potential pitfalls in doing research in a clinical setting. Yet, if you are well prepared, you may avoid most of these. As has been mentioned above, you need set a clear research question that you want to answer, plan your research design, plan your methodology, decide on your statistical techniques, and make sure you get ethical approval. One important way to ensure the research will be finished and finished correctly is to involve your colleagues. If you share your work with them, they are more likely to cooperate, identify study participants for you, and highlight problems you may not have considered. One exercise that you can do is to write an abstract of the research without the results. In doing this brief exercise, you can set out the methodology, subjects, research question, and statistical techniques.

One of the difficulties in conducting research in clinical areas occurs when there is an ethical conflict between using your clients for research and whether the research or the clinical needs take priority. One must always place clinical need above research interest. Sometimes it is better to undertake research and clinical work in different locations to keep your clinical interests and scientific interests apart

Choosing the right sampling technique is a crucial step that affects the whole study. If sampling is not done appropriately, the results may be flawed, irrespective of how well the study was conducted overall. When you are ready to start your research and wish to recruit study participants, you may find that there were many people who had the problem you are researching at the time you decided to do the research, but, once you start recruiting, they often seem scarce! This is a phenomenon noted by clinical researchers. Therefore, be prepared to go wider to recruit your participants by perhaps involving another agency or advising colleagues in similar facilities to yours that you are trying to recruit.

If you are running a trial with a control group, make sure that your control group is a genuine control and match the experimental group in every way possible, including matching by demographic features and definitions of the problem being researched. Too often, a reviewer on a journal will see that the control group does not match the experimental group and will reject an article on that basis

Another important aspect of doing your own research is the choice of appropriate data-collection tools. It is always better to choose standard, standardized, and well-recognized scales, questionnaires, and other types of measures as opposed to those developed on one's own.

It is not always easy to get research published. But there are some things you can do to increase your chances of getting an article accepted in a wellrespected journal. It is well to note the following points: 
- Scientific writing skills take a long time to acquire, and, with every article produced, these skills improve. Endurance and enthusiasm is the key. Also, collaboration with someone who already possesses these skills is encouraged.

- Scientific journal language is specific and differs among fields. For the beginner, it may be difficult and timely to write densely, specifically, and clearly. What may help is to read published articles to become familiar with the language style that is used and to ask someone experienced to "polish" the article.

- Scientific literature availability may be a problem for those working in smaller clinical facilities with smaller budgets. Their libraries simply may not be able to purchase access to journal full texts. You may want to invite for collaboration someone from an academic setting or a research facility with access to journal subscriptions. Also, you may ask the study authors for an author's copy. For more options how to search for scientific literature, see Chapter 7.

- Time between having completed the research and actually having an article accepted for publication may take months. The approximate time for receiving feedback from a journal is three months. Always try to plan ahead. You can save time by doing literature searches during the data-collection phase. Try to publish outcomes of the pilot phase of your research.

- Rejection of an article is common and every author has an experience of receiving negative feedback from the journal on his or her article. Always remember that most rejected articles may be improved based on the feedback that is usually sent together with the letter from the editorial office. Try to learn from the unsuccessful attempts, and do not allow pride or bitterness overcome you.

- Fighting frustration should be one of the skills you develop. Research and scientific publishing are very demanding, but getting your article published is very rewarding. All the pain pays off once you see your name connected with an important contribution to the field.

Chapters 7 and 8 in this book will help when you write up your work for publication. Read them carefully and follow the advice, because this will increase your chances of publication.

\section{Serving as a Reviewer}

Once you publish your first article, the chances increase that you will be asked by a journal to review someone else's manuscript. You may want to accept for the following reasons:

- reviewing an article will help you see and understand what the journal expects from authors (based, for example, on the reviewer guidelines and 
other requirements) and what the processes are inside the journal's "black box";

- reading a manuscript from a reviewer's position may help you adopt critical scientific thinking that you may later use when you write your own article; and

- reviewers are an "endangered species", and journal editors need competent, expert volunteers when arranging independent evaluation

\section{Conclusion}

Undertaking research as a clinician can be rewarding both for its intrinsic value and its ability to provide answers to many of those troubling clinical questions. It is also valuable for career development and can be an enhancement for a department or unit. It will take more time and effort but adds variety to the working week. You need to follow proper protocols, gain ethical approval, and obtain advice from colleagues or, if necessary, a local academic department. Do not try to take short cuts or believe that, because you are working in the reality of the treatment setting, you know better than academics. Nothing is worse than spending a lot of time and effort on a project for it then failing because of a lack of thorough preparation. Good luck with your research!

\section{Acknowledgements}

We offer many thanks to Richard Saitz for his helpful suggestions in our writing of this chapter.

Please visit the website of the International Society of Addiction Journal Editors (ISAJE) at www.isaje.net to access supplementary materials related to this chapter. Materials include additional reading, exercises, examples, PowerPoint presentations, videos, and e-learning lessons.

\section{Note}

1 The widely advertised evidence-based approach is viewed by some as too narrow and formalistic. Hjørland (2011) promotes what is called researchbased practice that, besides taking into account quantitative approaches, also considers as legitimate theoretical work and qualitative research. 


\section{References}

Carnwath, T., Garvey, T., \& Holland, M. (2002). The prescription of dexamphetamine to patients with schizophrenia and amphetamine dependence. Journal of Psychopharmacology, 16, 373-377. DOI: https://doi. org/10.1177/026988110201600414

Edwards, G. (in preparation). Seeing Addiction (Book).

Edwards, G., \& Babor, T. F. (Eds.). (2012). Addiction and the making of professional careers. New Brunswick, NJ: Transaction Publishers.

Hjørland, B. (2011). Evidence based practice: An analysis based on the philosophy of science. Journal of the American Society for Information Science and Technology, 62, 1301-1310. DOI: https://doi.org/10.1002/asi.21523

Jowett, S. M., Macleod, J., Wilson, S., \& Hobbs, F. D. R. (2000). Research in primary care: Extent of involvement and perceived determinants among practitioners from one English region. British Journal of General Practice, 50, 387-389.

Miovský, M., Čablová, L., Kalina, K., \& Štastná, L. (2014). The effects of ADHD on the course and outcome of addiction treatment in clients of therapeutic communities: Research design. Adiktologie, 14, 392-400.

Pates, R. M., \& Gray, N. (2009). The development of a psychological theory of needle fixation. Journal of Substance Use, 14, 312-324. DOI: https://doi. org/10.3109/14659890903235876

Pates, R. M., McBride, A. J., Ball, N., \& Arnold, K. (2001). Towards an holistic understanding of injecting drug use: An overview of needle fixation. Addiction Research \& Theory, 9, 3-17. DOI: https://doi. org/10.3109/16066350109141769

Salmon, P., Peters, S., Rogers, A., Gask, L., Clifford, R., Iredale, W., Dowrick C., \& Morriss, R. (2007). Peering through the barriers in GPs' explanations for declining to participate in research: The role of professional autonomy and the economy of time. Family Practice, 24, 269-275. DOI: https://doi.org/10.1093/ fampra/cmm015

Storbjörk, J. (2014). Implications of enrolment eligibility criteria in alcohol treatment outcome research: Generalisability and potential bias in 1- and 6-year outcomes. Drug and Alcohol Review, 33, 604-611. DOI: https://doi. org/10.1111/dar.12211 
